Revista

Multi-Ensayos

Vol. 5, $\mathrm{N}^{\circ} 9$

ISSN: 2412-3285

https://multiensayos.unan.edu.ni

DOI: https://doi.org/10.5377/multiensayos.v5i9.9429

\title{
Las experiencias de aprendizaje el mejor gusto de la docencia
}

\section{Learning experiences the best taste of teaching}

Norman Rafael López Sánchez ${ }^{1}$

\section{RESUMEN}

La experiencia es el resultado de todo trabajo y especialmente como docente la mejor satisfacción es el nuevo aprendizaje que se lleva día a día desde las aulas de clase, el poder compartir con los estudiantes, el gusto de saborear una nueva forma de aprender, son aspectos que hacen de la docencia un formador para el presente y el futuro, capaces de enfrentar desafíos que hoy en día la sociedad demanda, adaptados a los avances de la ciencia y la tecnología, es por eso que en el presente ensayo se realiza con el fin de analizar algunas de las experiencias más relevantes vividas en el desarrollo del proceso de formación, las cuales permiten una vía para la transformación en el ámbito educativo.

Palabras claves: docencia; estudiantes; aprendizaje; experiencia; transformación.

\section{ABSTRACT}

The experience is the result of all work and especially as a teacher the best satisfaction is the new learning that takes day by day from the classrooms, the ability to share with students, the taste of a new way of learning, are aspects that They make teaching a former for the present and the future, capable of facing challenges that nowadays the society demands, adapted to the advances of the science and the technology, that is why in the present essay is carried out in order to analyze some of the Experience more relevant in the development of the training process, which allow a pathway for transformation in the educational field.

Keywords: teaching; students; learning; experience; transformation.

1 Docente de UNAN-Managua/FAREM-Estelí. Correo electrónico: lopeznorman88@gmail.com (C) 2019 Revista Multi-Ensayos. 


\section{INTRODUCCIÓN}

Según (Arana, 2007:3) expresa que el mejor gusto de todo docente es estar día con día en el aula compartiendo con una experiencia mutua de aprender y, al paso del tiempo, encontrar estudiantes que exclamen con gusto "iProfe, usted me dio clases!"

Así mismo el gusto por la docencia es enseñar a los jóvenes a pensar, a tener criterio, a decidirse por una opción, a utilizar los diferentes enfoques sociales y científicos para crear el futuro, desarrollar inquietudes, formar carácter y valores personales entre los estudiantes para su futuro profesional. Descubrir, curso a curso, nuevas formas de detonar las potencialidades de los estudiantes (Vargas, 2010)

\section{DESARROLLO}

Hay tantos jóvenes en el país y especialmente en la Región Norte que es estimulante saber que se les puede enseñar algo, no solamente relacionado con el aprendizaje, sino con su desarrollo en la sociedad. Surge así la satisfacción ante el reconocimiento de los estudiantes por haber logrado en ellos el aprendizaje, la comprensión; por haber colaborado en el cambio de su perspectiva de vida.

Es aquí donde el trabajo del docente se centra en formar jóvenes con las herramientas y elementos necesarios que le permitan enfrentarse a la vida laboral, donde las experiencias deben de ser tangibles desde el inicio, es decir que no sean fantasías que como resultado final se puede obtener la desilusión del actor principal que es el estudiante. (Vezub, 2007)

Así mismo, como un primer año de experiencia en asumir el reto de formar profesionales para un futuro, preparándolos técnica y científicamente en personas competentes capaces de enfrentar las necesidades que hoy en día la sociedad demanda.

Donde ser parte de la docencia universitaria de la Universidad Nacional Autónoma de Nicaragua (FAREM- Estelí), representa el punto de partida al asumir el reto de una preparación activa y permanente comprometida con el desarrollo de nuestra nación y el prestigio de la misma.

Donde se desarrollaron los cursos de Matemática General, Estadística Descriptiva y Estadística I, para las cuales lo primordial fue su aplicación en la vida cotidiana desarrollando técnica y métodos que permitieran al estudiantado obtener un aprendizaje significativo, tomando en cuenta en todo momento la importancia de su carrera, generando de esta manera una participación activa.

Otro aspecto importante que se vivió fue la .convivencia con los estudiantes, la cual es extraordinariamente interesante y vital, aunque nunca deja de ser profesional desde un saludo amable, un recorrido por el aula y una pregunta personal, una preocupación por su estado afectivo y emocional (¿cómo estás?, ¿por qué no has venido?, ¿cómo te sientes?, ¿desayunaste?, donde es notable que invertir de tres a cinco minutos, se obtiene disposición por parte de los educandos, permitiendo una interacción bilateral entre docente-estudiante.

Por ende, el acercamiento a los estudiantes genera un ambiente amable, que facilita el estudio, la confianza para decir lo que se piensa y por ambas partes se establece la importancia del cumplimiento de compromisos exigidos por el curso, así mismo la comunicación activa. (Novak, 1995) 
Es donde vemos que la experiencia es un sinónimo de acción, compromiso, reproducción, motivación, todo esto para lograr un objetivo: un aprendizaje significativo que comprendan por qué la acción llevada a cabo y no por una simple mecanización como muchas veces suele suceder.

Por lo cual, es importante el respeto y la verdad, escuchar con atención, buscar la empatía con su manera de pensar, recordar que son aptos siempre y en todo momento, invitarlos a descubrir que son capaces de hacer cosas, aprender lo importante relacionándolo con su vida cotidiana. (Jiménez, 2011) Otra de las experiencias fue encontrarse con estudiantes de distintos ritmos de aprendizaje, unos interesados y otros desinteresados por asumir responsabilidades, donde se trabajó por la promoción de valores, permitiendo al estudiantado generar ambientes de reflexión, recordando constantemente la misión y visión de la universidad.

Por eso es el momento donde se debe quedar bien claro que una sola experiencia de aprendizaje satisfaga las necesidades de un grupo de estudiantes, puesto que existe una diversidad de estilos para aprender, es decir, no todos van alcanzar el mismo grado de conocimiento.

Es así que cuando diseñamos la experiencia de aprendizaje debemos de buscar siempre lo mejor, lo atractivo, lo novedoso, la innovación, todo esto debe ser constante en todo tiempo, que deje al estudiante impactado, logrando una comprensión de lo vivido, queremos dar todo lo que construya, aunque a veces no se logre lo que deseamos poniendo en práctica solo el que no arriesga no pierde, pero reforzándolo siempre y animándose con el que persevera alcanza.

Por otra parte, en el desarrollo de todo proceso de aprendizaje se obtienen rendimiento académicos satisfactorios, buenos, excelentes, otros en el que se debe mejorar, donde lo excelente se logra con el compromiso, entrega, dedicación animándose siempre en no dejar de aprender, para lo cual, necesita y requiere límites, disciplina y libertad, lo cual parece sencillo a nivel de descripción, pero no de acción. Es así como se logra con una experiencia honesta, no engañar al educando con prestaciones muy bonitas de efectos y colores llamativos para que vea que si se sabe aprovechar la tecnología, aunque muchas veces son ellos mismos quienes los diseñan, olvidándonos de esta manera el objetivo que buscamos que es siempre el aprendizajes significativo.

\section{CONCLUSIÓN}

Lo que se ha venido planteando son algunas de las experiencias que más incidieron en los desarrollos de los distintos cursos, además permitirán prepararse para los nuevos retos venideros, y a los distintos cambios que experimentan hoy en día la ciencia y la tecnología.

Lo más importante de este modelo es formar profesionales analíticos, críticos, observadores, que comprendan, que razonen, que construyan su propio conocimiento, sin dejar al lado que la experiencia del aprendizaje se da durante toda la vida y en los diferentes contextos del entorno.

Todo lo vivido en un proceso implica trabajo, pero la mayor satisfacción es compartir y aprender, saber que cada día aportas al crecimiento de otras personas, por lo cual se puede decir "hoy somos más que ayer, pero no más que mañana", es decir que cada día se aprende algo nuevo, sin desanimarse al estar atento a lo que está pronto por llegar. 


\section{REFERENCIAS}

Jiménez, G. \&. (2011). Congreso de educación en México. Una Perspectiva Epistemológica de la Formación Docente.

Novak, J. (1995). El uso de herramientas metacognitivas para facilitar el aprendizaje significativo y la construcción de conocimientos. Estudios de pedagogía y psicología, 159- 181.

Vargas, L. (2010). Congreso de Educación. Metas 2021 en la formación docente. Buenos Aires.

Vezub, L. (2007). La formación y el desarrollo profesional docente frente a los nuevos desafíos de la escolaridad. PROFESORADO: Revista de curriculum y Docencia, 18. 